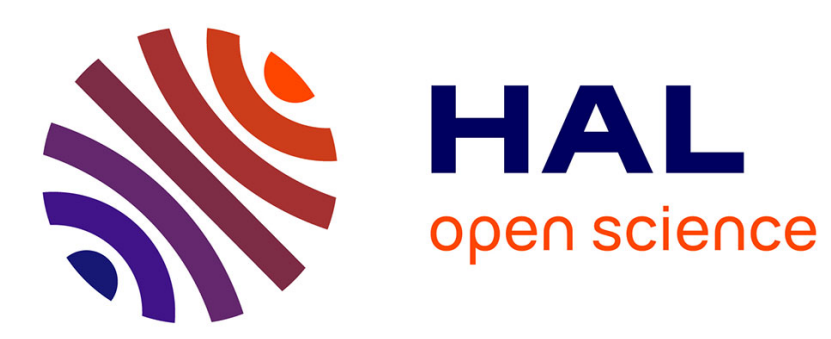

\title{
MASS EFFECT ON THERMODIFFUSION USING MOLECULAR DYNAMICS
}

Guillaume Galliero, Mathilde Bugel, Bernard Duguay, François Montel

\section{To cite this version:}

Guillaume Galliero, Mathilde Bugel, Bernard Duguay, François Montel. MASS EFFECT ON THERMODIFFUSION USING MOLECULAR DYNAMICS. Journal of Non-Equilibrium Thermodynamics, 2007, 32 (3), pp.251-258. 10.1515/JNETDY.2007.017 . hal-00295060

\section{HAL Id: hal-00295060 \\ https://hal.science/hal-00295060}

Submitted on 11 Jul 2008

HAL is a multi-disciplinary open access archive for the deposit and dissemination of scientific research documents, whether they are published or not. The documents may come from teaching and research institutions in France or abroad, or from public or private research centers.
L'archive ouverte pluridisciplinaire HAL, est destinée au dépôt et à la diffusion de documents scientifiques de niveau recherche, publiés ou non, émanant des établissements d'enseignement et de recherche français ou étrangers, des laboratoires publics ou privés. 


\title{
MASS EFFECT ON THERMODIFFUSION USING MOLECULAR DYNAMICS
}

\author{
Guillaume GALLIERO ${ }^{1 *}$, Mathilde BUGEL ${ }^{2}$, Bernard DUGUAY ${ }^{3}$ and François MONTEL ${ }^{4}$ \\ ${ }^{1}$ Laboratoire LETEM, Université de Marne-la-Vallée, Bât. Lavoisier, Champs-sur-Marne, F- \\ 77454 Marne-la-Vallée Cedex 2, FRANCE. \\ ${ }^{2}$ Laboratoire TREFLE, UMR 8508, Université Bordeaux I, ENSCPB, 16 Avenue Pey- \\ Berland, F-33607 Pessac Cedex, FRANCE. \\ ${ }^{3}$ Laboratoire LPCM, UMR 5803, Université Bordeaux I, 351 Cours de la Libération, F-33405 \\ Talence Cedex, FRANCE. \\ ${ }^{4}$ TOTAL, CSTJF, Avenue Larribau, F-64018 Pau, FRANCE. \\ *Corresponding author: galliero@univ-mlv.fr
}

\begin{abstract}
The scope of this study is to improve the understanding of the thermal diffusion process on a microscopic scale by studying the mass effect on thermal diffusion factors. To achieve such a goal, Non Equilibrium Molecular Dynamics simulations are performed on binary mixtures of simple Lennard-Jones spheres for a large range of thermodynamic states. Mixtures for which only the mass between species differs, up to mass ratios of 50, are analyzed (isotope like mixtures). In equimolar mixtures, it is shown that the link between the thermal diffusion factors and the ratio between the difference in masses and the sum of masses holds approximately for all states studied. In addition, it is found that this link strongly depends on density but weakly on temperature. In nonequimolar mixtures, results indicate that the effect of mass ratio between species depends on the molar fraction. Using the data computed, a simple density dependent correlation is proposed to quantify the mass effect in Lennard-Jones binary mixtures. Finally, it is shown that, taking into account only the mass effect, this correlation is able to provide a reasonable estimation of thermodiffusion in $n$-pentane $/ n$ decane mixtures which underlines intrinsic weakness of some of the usual thermodynamic models predicting thermodiffusion.
\end{abstract}




\section{INTRODUCTION}

A precise knowledge of the thermodiffusion coefficients in hydrocarbon mixtures is required in the oil\&gas industry to ensure reliable formation fluid evaluation. Given the huge number of components in these mixtures, the use of simple and efficient models is required. For such systems, Molecular Dynamics (MD) reveals itself to be a useful tool to improve our understanding of the physical processes that govern thermodiffusion [1-5], which is a compulsory step in order to build reliable correlations on it or to improve existing models.

Among the effects that might affect thermal diffusion amplitude is the mass ratio between the species [1-3, 6-7]. Quite surprisingly some of the usual thermodynamic models predicting thermodiffusion [8-10] do not take into account an explicit contribution of the mass effect apart from the one at low density. For example, the Kempers model [8] applied to an ideal binary solution (such as an isotope mixture) yields a thermal diffusion factor, $\alpha_{\mathrm{T}}$, that reduces to its low density contribution whatever the thermodynamic state (see for instance section III.G of Kempers paper [8]) which is certainly not correct in dense systems.

In this work, an attempt is made to quantify the mass effect on thermodiffusion for a wide range of conditions by studying isotope like mixtures (i.e. ideal solutions for which only mass differs between species). To do so, Non Equilibrium Molecular Dynamics simulations (NEMD) have been performed on binary mixtures of Lennard-Jones 12-6 (LJ) spheres. A wide range of mass ratios $\left(m_{2} / m_{1}\right.$ ranging from 2 to 50$)$ and various thermodynamic conditions (from low to high densities and sub to super critical temperatures) have been explored. Then, using these results, a simple correlation has been constructed to quantify the mass effect and has been applied on a real $n$-alkane mixture.

\section{SIMULATION DETAILS}

Simulations are performed on systems composed of 1500 spheres interaction through a LJ potential whose molecular parameters are $\sigma=3.723 \AA$, $\varepsilon=1177 \mathrm{~J} / \mathrm{mol}$ and $m=0.016 \mathrm{~kg} / \mathrm{mol}$. 
The LJ potential has been truncated at $2.5 \sigma$. The Verlet velocity algorithm and periodical boundary conditions are used. As long as the LJ potential follows the corresponding states law, reduced thermodynamic variables $\left(\rho^{*}=N \sigma^{3} V^{-1}\right.$ and $T^{*}=k_{B} T \varepsilon^{-1}$, where $N$ is the number of particles, $V$ the volume of the simulation box and $T$ the average temperature) are used [3]. To compute the thermal diffusion factors, a boundary driven NEMD algorithm proposed by Hafskjold [1] is used. After equilibration, a reduced heat flux, $J_{q}^{*}=J_{q} \sigma^{3} m^{1 / 2} \varepsilon^{-3 / 2}$, of $0.1-0.3$ is applied to the system during $7.510^{6}$ nonequilibrium time steps $\left(\delta t^{*}=\delta t \varepsilon^{1 / 2} m^{-1 / 2} \sigma^{-1}=0.002\right)$. Further details can be found in [3]. The statistical errors associated to the thermal diffusion factor are around $+/-5 \%$, except in very dense phase where errors reach $10 \%$, they are omitted in the following.

\section{RESULTS}

In low density systems, kinetic theories are efficient. So, as a starting point to construct a correlation on the mass effect, a kinetic theory formulation of the thermal diffusion factor, $\alpha_{T}$, has been used. In this frame, for an isotope binary mixture [7]:

$$
\alpha_{T}=\alpha_{0} \frac{\left(m_{2}-m_{1}\right)}{\left(m_{2}+m_{1}\right)}\left(1-\gamma\left(x_{2}-x_{1}\right) \frac{\left(m_{2}-m_{1}\right)}{\left(m_{2}+m_{1}\right)}\right)
$$

where $x_{i}$ and $m_{i}$ are the molar fraction and the molecular weight of compound $i$ and $\alpha_{0}$ and $\gamma$ are coefficients which are a function of the thermodynamic state only. The idea is to use MD simulations to check the validity of such a relation for other conditions (dense systems, large mass ratios between species).

\subsection{Mass effect in equimolar binary systems}

In equimolar mixtures eq. (1) reduces to $\alpha_{T}=\alpha_{0}\left(m_{2}-m_{1}\right) /\left(m_{2}+m_{1}\right)$. So, in order to verify such a relation, five different mass ratios $\left(m_{2} / m_{1}\right.$ ranging from 2 to 50) have been tested for eighteen thermodynamic states $\left(T^{*}\right.$ ranging from 1 to 2.5 with a step of 0.5 and $\rho^{*}$ being equal 
to $0.3,0.5,0.7,0.8$ and 0.9$)$. These reduced thermodynamic conditions cover systems from low to high densities and sub to supercritical temperatures, (the critical point of the LJ fluid is located at $\rho_{c}^{*} \approx 0.32$ and $T_{c}^{*} \approx 1.3$ ). Points in the two-phases region have been discarded.

Figure 1 shows that $\alpha_{T}$ increases with mass ratio in all cases and depends on the thermodynamic conditions (strongly on $\rho^{*}$ but weakly on $T^{*}$ ). In addition, it has been found that eq. (1) is approximately respected; i.e. for a given thermodynamic state, $\alpha_{0}$ is weakly dependent on mass ratio. Nevertheless, it should be noted that for $m_{2} / m_{1}=2$ and 50 values of $\alpha_{0}$ are generally higher than for average mass ratios, $m_{2} / m_{1}=5,10$ and 20 as can be deduced from figure 1. To provide a general trend of the results, an average of the $\alpha_{0}$ for each thermodynamic state, noted $\alpha_{0 \_a v}$, has been calculated using the results obtained for the five mass ratios tested, see figure 2 .

Figure 2 shows that $\alpha_{0 \_ \text {av }}$ has a strong dependence on density (monotone increase) but a weak one on temperature. So, a correlation only based on density dependence is proposed:

$$
\alpha_{0 \_a v}=-4.2\left(\rho^{*}\right)^{4}+6.6\left(\rho^{*}\right)^{2}+0.55
$$

This relation, combined with eq. (1), yields for $\alpha_{T}$ a satisfying Average Absolute Deviation (AAD), compared to NEMD results, of $5.2 \%$ with a maximum of $19.1 \%$. It should be noted that this correlation is not valid for $\rho^{*} \rightarrow 0$, for which kinetic theories relation holds [7].

\subsection{Nonequimolar binary mixtures}

To study the behaviour of eq. (1) in nonequimolar systems, simulations have been performed for five different molar fractions, $x_{1}=0.1,0.25,0.5,0.75$ and 0.9 . Four thermodynamic states and two mass ratios have been explored, see figure 3.

Results given on figure 3 show that, in all cases, the mass effect on thermodiffusion is dependent on the molar fraction (i.e . $\gamma$, from eq. (1), is not equal to zero). $\alpha_{\mathrm{T}}$ increases (nearly linearly) with the molar fraction of the lightest component, which is consistent with previous 
findings [3]. In addition, the increase with the molar fraction depends on the thermodynamic state and is the largest in the liquid state $\left(T^{*}=1, \rho^{*}=0.7\right)$. Eq. (1) is able to correlate reasonably the results if $\gamma$ varies from 0.25 to 0.5 (which is plausible compared to the values given in [7]) depending on the thermodynamic state. Nevertheless, the results are insufficient to construct a $T^{*}, \rho^{*}$ dependent correlation for $\gamma$. Moreover, simply by taking

$$
\gamma=0.35
$$

a correct estimation of $\alpha_{\mathrm{T}}$ can be reached knowing $\alpha_{0}(\mathrm{AAD}=3.51 \%)$.

Using the previous findings, the correlation scheme proposed, eq.(1) combined with eqs.(2) and (3), has been applied on the 114 points of simulations, given in figures 1 and 3. This correlation yields an overall $\mathrm{AAD}=6.4 \%$ with a maximum of $19.1 \%$, which is satisfying compared to the large variety of conditions tested and the inherent approximations and uncertainties.

\subsection{Application to a real mixture.}

It is believed that the correlation scheme proposed, eqs. (1-3), is able to provide a reasonable estimation of the thermal diffusion factors in normal alkane mixtures. Even if real $n$-alkane are far from being LJ spheres, such assumption is supported by the fact that, on methane/ndecane mixtures Simon et al. [11] have shown that results on thermodiffusion using LJ spheres and multicenter flexible molecular models are similar (which is not the case for thermal conductivities). In addition, when $n$-alkanes are modelled through LJ spheres, size and energy parameters contributions to thermodiffusion (which are opposite [12]) tend to compensate each other if the two $n$-alkanes are not too dissimilar.

As a test, the correlation has been applied on $n$-pentane/n-decane $\left(\mathrm{C}_{5}-\mathrm{C}_{10}\right)$ mixtures, which have been studied experimentally in the liquid state at ambient pressure and $T=300 \mathrm{~K}$ [13]. It should mentioned that to apply such a correlation, one has to estimate the reduced density of the mixture, $\rho_{r}=\rho / \rho_{c}$, where $\rho_{c}$ is the critical density of the mixture and then use the fact that 
$\rho_{c}^{*} \approx 0.32$, which implies that $\rho^{*}=0.32 \rho_{r}$. To estimate the critical density of each mixture Kay's rules have been applied [14].

Table 1 shows that, on this particular mixture, the correlation works well, which tends to show that in such mixtures the mass effect is certainly the predominant one (over size and energetic effects). Nevertheless, keeping in mind the intrinsic uncertainties of the correlation and the crude model chosen, the good result of the correlation proposed on the $\mathrm{C}_{5}-\mathrm{C}_{10}$ mixtures should not hide the fact that, in this case, different errors certainly compensate each other. Hence, to be applicable to a larger range of mixtures, an improvement of such an approach must include more refinement (inertia momentum effects, non ideal effects, ...).

Besides, the fact that the mass effect is certainly the predominant one in some mixtures raises the question of the efficiency of some of the usual thermodynamic models [8-10] (for which the mass effect is not explicitly taken into account) to predict thermodiffusion in such mixtures. This result may partly explain why, despite some noticeable progresses, these models experienced some troubles to predict thermodiffusion even in simple systems [8-10, 14]. We could imagine that, with an appropriate modification of these models to better represent the mass effect, improved predictions could be reached.

\section{CONCLUSION}

In this study, a Boundary Driven NonEquilibrium Molecular Dynamics algorithm has been applied on binary mixtures of simple Lennard-Jones spheres in order to compute the thermal diffusion factors for a wide range of thermodynamic states. Mixtures in which only the mass between species differs (isotope like) have been analysed. Mass ratios ranging from 2 to 50 have been studied. Then, these results have been used to develop a simple correlation to quantify the mass effect in such mixtures.

In a first part, it is shown on equimolar systems that the linear relation between the thermal diffusion factors and the difference in masses divided by the sum of masses holds 
approximately for all conditions studied. In addition, the coefficient that appears in this relation, $\alpha_{0}$, is shown to be strongly dependent on density but weakly on temperature. Hence, a simple density dependent correlation is proposed to quantify $\alpha_{0}$ in LJ systems, eq. (2).

In a second part, simulations on nonequimolar mixtures show that $\alpha_{T}$ is dependent on molar fraction (increase with the molar fraction of the lightest compound in a fairly linear way), whatever the thermodynamic state and the mass ratio. In addition, it appears that eq. (1) is able to provide a good estimation of the dependence of $\alpha_{T}$ with the molar fraction, when taking simply $\gamma=0.35$. Thus, by combining eq. (1) with eqs. (2-3), it is possible to quantify the thermodiffusion in LJ isotope like systems for all conditions, the AAD on the 114 points simulated being equal to $6.4 \%$ with a maximum below $20 \%$.

Finally, it is shown that this correlation, compared to experiments, provide consistent results on a $\mathrm{C}_{5}-\mathrm{C}_{10}$ mixture, which tends to show the predominant effect of mass on thermodiffusion in such systems. In addition, this result highlights an intrinsic weakness of some of the existing thermodynamic models to predict thermodiffusion which do not take into account explicitly this mass effect except the low density contribution.

\section{ACKNOWLEDGEMENTS}

We thank the CINES and the SIMOA, which provided computer time required for this study and the ESA that funds this study under the DSC project.

\section{REFERENCES}

[1] B. Hafskjold, T. Ikeshoji, S. Ratkje, On the molecular mechanism of thermal diffusion in liquids, Mol. Phys., 80 (1993), 1389-1412.

[2] D. Reith, F. Müller-Plathe, On the nature of thermal diffusion in binary Lennard-Jones liquids, J. Chem. Phys., 112 (2000), 2436-2443.

[3] G. Galliero, Thermal diffusion in Lennard-Jones fluids in the frame of the law of the corresponding states, Fluid Phase Equi., 224 (2004), 13-22. 
[4] B. Rousseau, C. Nieto-Draghi, J.B. Avalos, The role of molecular interactions in the change of sign of the Soret coefficient, Europhys. Letters, 67 (2004), 976-982.

[5] S; Yegani, M. D. Ganji, Dependence of thermal diffusion factor of binary mixtures to the thermodynamic state by NEMD simulation, Chem. Phys., 318 (2005), 171-179.

[6] G. Wittko, W. Köhler, Universal isotope effect in thermal diffusion of mixtures containing cyclohexane and cyclohexane- d12, J. Chem. Phys., 123 (2005), 014506 -6.

[7] J.M. Kincaid, E.G.D. Cohen, M. Lopez de Haro, The Enskog theory for multicomponents mixtures. IV. Thermal diffusion, J. Chem. Phys., 86 (1986), 963-975.

[8] L.J.T.M Kempers, A comprehensive thermodynamic theory of the Soret effect in a multicomponent gas, liquid, or solid, J. Chem. Phys., 115 (2001), 6330-6341.

[9] K. Shukla, A. Firoozabadi, A new model of Thermal diffusion coefficients in binary hydrocarbon mixtures, Ind. Eng. Chem. Res., 37 (1998), 3331-3342.

[10] M.M. Gonzalez-Bagnoli, A.A. Shapiro, E.H. Stenby, Evaluation of the thermodynamic models for the thermal diffusion factor., Phil. Mag., 17-18 (2003), 2171-2183.

[11] J.M. Simon, B. Rousseau, D.K. Dysthe, B. Hafskjold, Thermal diffusion in methane/ndecane mixtures by molecular dynamics using spherical and flexible multicenter models, Entropie, 217 (1999), 29-32.

[12] G. Galliero, B. Duguay, J.-P. Caltagirone, F. Montel, Thermal diffusion sensitivity to the molecular parameters of a binary equimolar mixture, a nonequilibrium molecular dynamics approach, Fluid Phase Equi. 208 (2003), 171-188.

[13] A. Perronace, C. Leppla, F. Leroy, B. Rousseau, S. Wiegand, Soret and mass diffusion measurements and molecular dynamics simulations of $n$-pentane $/ n$-decane mixtures, $J$. Chem. Phys., 116 (2002), 3718-3729.

[14] B.E. Poling, J.M. Prausnitz, J.P. O'Connell, The properties of gases and liquids, McGraw-Hill, 2001. 
Table:

Table $1: \alpha_{\mathrm{T}}$ in $\mathrm{C}_{5}-\mathrm{C}_{10}$ liquid mixtures for various molar fractions. Comparison between experiments [8] and correlation results, eqs. (1-3).

\begin{tabular}{|c|c|c|}
\hline $\mathrm{x}_{\mathrm{C} 5}$ & Experiments & Correlation \\
\hline 0.2 & $1.14 \pm 0.02$ & 0.91 \\
\hline 0.5 & $0.99 \pm 0.01$ & 1.01 \\
\hline 0.8 & $1.05 \pm 0.02$ & 1.10 \\
\hline
\end{tabular}


Figures :

Figure 1: Thermal diffusion factors in binary equimolar mixtures for different mass ratios and various thermodynamic conditions. Circles correspond to $\rho^{*}=0.3$, down triangles to $\rho^{*}=0.5$, squares to $\rho^{*}=0.7$, diamonds to $\rho^{*}=0.8$ and up triangles to $\rho^{*}=0.9$. Black symbols correspond to $T^{*}=1.0$, dark grey ones to $T^{*}=1.5$, grey ones to $T^{*}=2.0$ and open symbols to $T^{*}=2.5$.

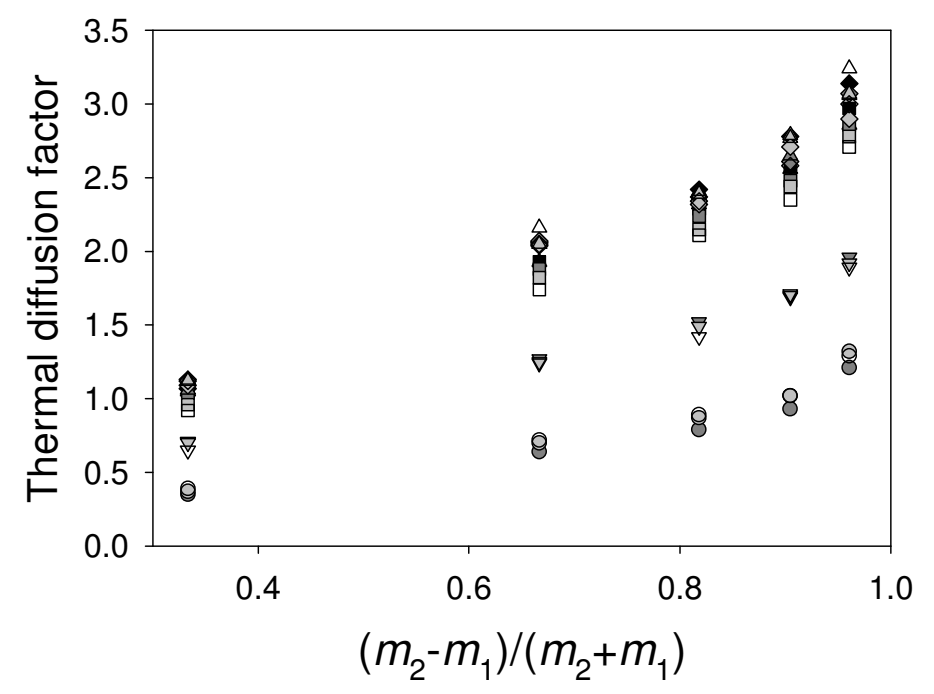


Figure $2: \alpha_{0 \_a v}$ versus density for various temperature.

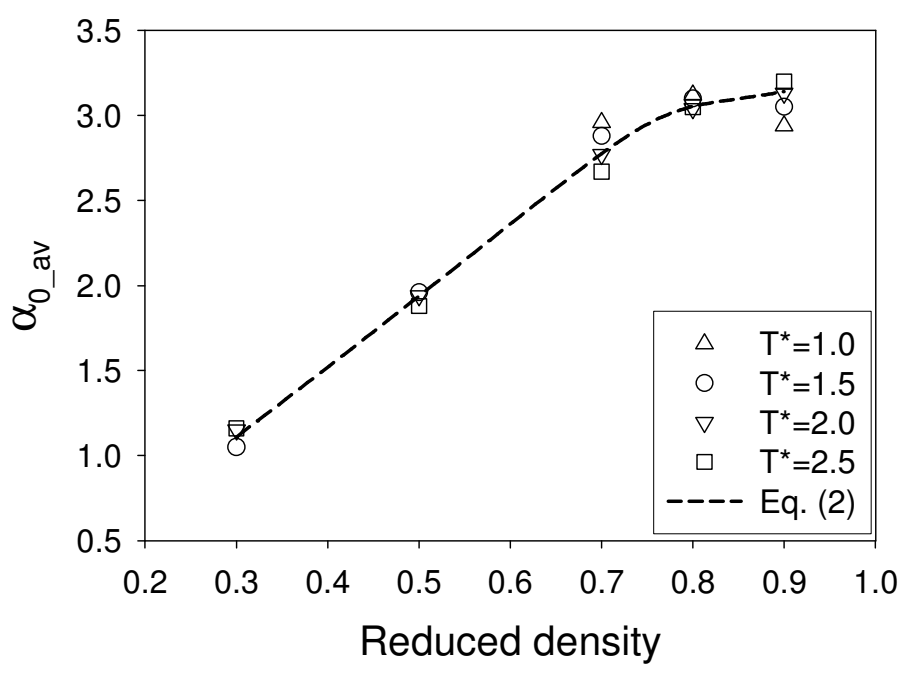


Figure 3: Thermal diffusion factors versus molar fraction for various conditions. Circles correspond to $T^{*}=1.5, \rho^{*}=0.3$ and $m_{2} / m_{1}=5$, down triangles to $T^{*}=1.5, \rho^{*}=0.3$ and $m_{2} / m_{1}=10$, squares to $T^{*}=2.5, \rho^{*}=0.3$ and $m_{2} / m_{1}=10$, diamonds to $T^{*}=1, \rho^{*}=0.7$ and $m_{2} / m_{1}=10$, up triangles to $T^{*}=2.5, \rho^{*}=0.7$ and $m_{2} / m_{1}=5$ and hexagons to $T^{*}=2.5, \rho^{*}=0.7$ and $m_{2} / m_{1}=10$.

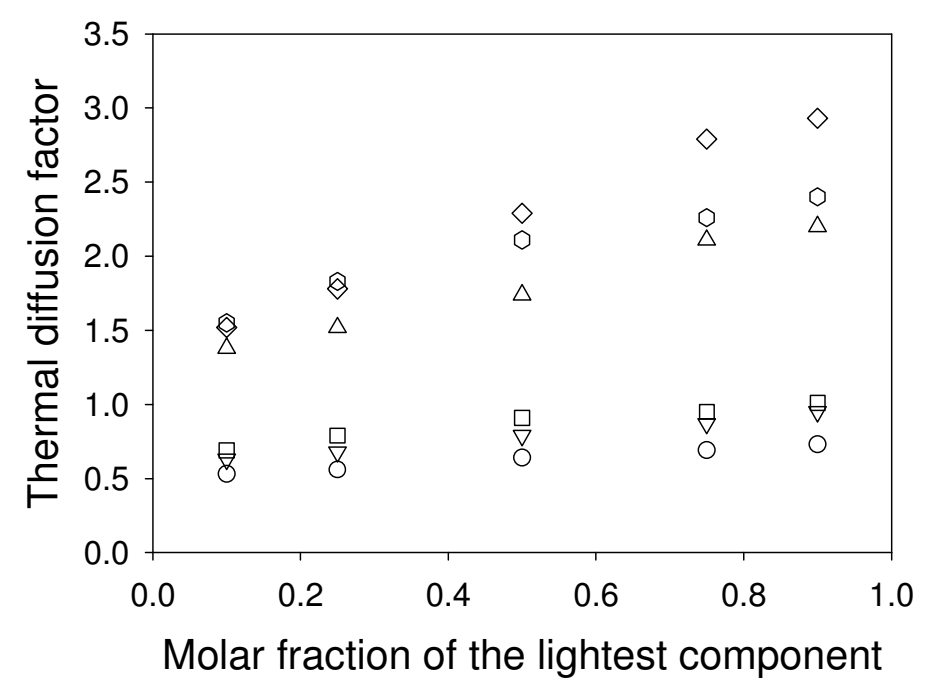

